\title{
ABC of chronic obstructive pulmonary disease Diagnosis
}

\author{
Graeme P Currie, Joe S Legge
}

As with most other common medical conditions, the diagnosis of chronic obstructive pulmonary disease (COPD) depends on a consistent history and appropriate examination findings. Confirmatory objective evidence is provided by spirometry. Doctors should consider the possibility of COPD in any patient aged 35 years or older with any relevant respiratory symptom and a history of smoking.

\section{History}

Consider COPD in any current or former smoker age $>35$ years who complains of any combination of breathlessness, chest tightness, wheeze, sputum production, cough, frequent chest infections, and impaired exercise tolerance.

COPD may also be present in the absence of noticeable symptoms, so look for it in individuals who are current or former smokers. Pay particular attention to features in the history or examination that may suggest an alternative or concomitant diagnosis. Since asthma tends to be the main condition in the differential diagnosis of COPD, a careful history should be taken in order to help distinguish between the disorders. Ask about previous and present occupations, particularly with regard to exposure to dusts and chemicals.

Record the patient's current smoking status and calculate the number of smoking pack years.

- Number of pack years $=$ (number of cigarettes smoked/day $\times$ number of years smoked) $/ 20$

- For example: a patient who has smoked 15 cigarettes a day for 40 years has a $(15 \times 40) / 20=30$ pack year smoking history.

Calculating the number of smoking pack years overcomes the problems of differences in duration and intensity of cigarette smoking. The decline in forced expiratory volume in 1 second $\left(\mathrm{FEV}_{1}\right)$ is generally related to the extent of cumulative exposure, although there is wide variability between individuals.

As part of the overall assessment of COPD, find out about symptoms of anxiety and depression, other medical conditions, current drug treatments, frequency of exacerbations, previous hospitalisations, exercise limitation, number of days missed from work and their financial impact, and extent of social and family support. COPD causes systemic effects, with weight loss being an under-recognised symptom in advanced disease.

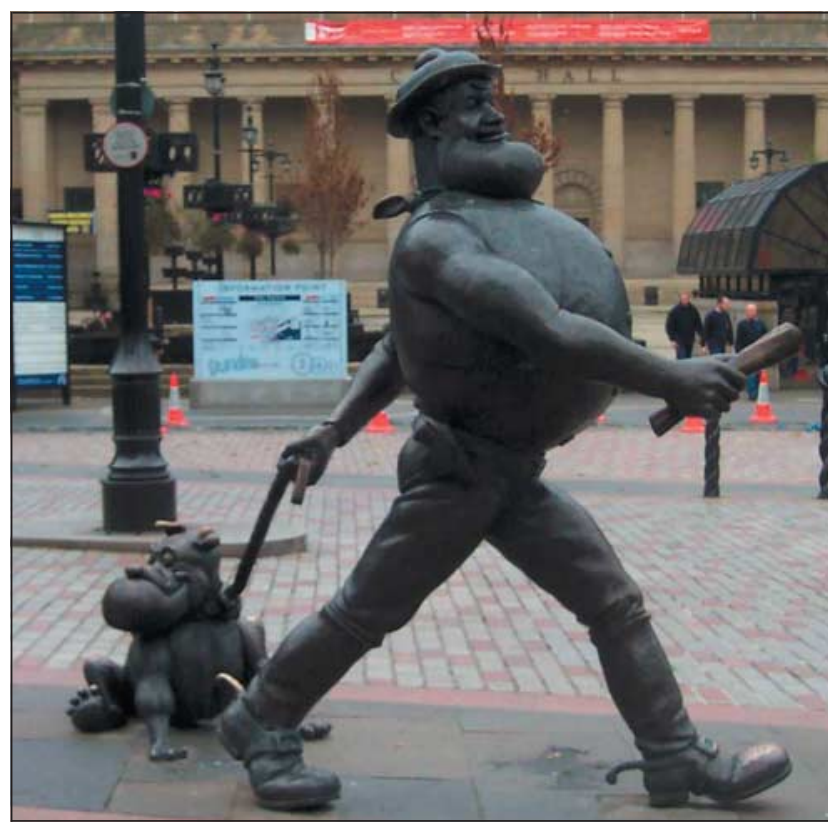

Patients with advanced COPD can develop an increased anteroposterior chest diameter-a barrel shaped chest

\begin{tabular}{|c|c|c|}
\hline \multicolumn{3}{|c|}{ Differences between COPD and asthma } \\
\hline & COPD & Asthma \\
\hline Age & $>35$ years & Any age \\
\hline Cough & Persistent and productive & $\begin{array}{l}\text { Intermittent and } \\
\text { non-productive }\end{array}$ \\
\hline Smoking & Almost invariable & Possible \\
\hline Breathlessness & Progressive and persistent & $\begin{array}{l}\text { Intermittent and } \\
\text { variable }\end{array}$ \\
\hline Nocturnal symptoms & $\begin{array}{l}\text { Uncommon unless in } \\
\text { severe disease }\end{array}$ & Common \\
\hline Family history & $\begin{array}{l}\text { Uncommon unless family } \\
\text { members also smoke }\end{array}$ & Common \\
\hline $\begin{array}{l}\text { Concomitant eczema } \\
\text { or allergic rhinitis }\end{array}$ & Possible & Common \\
\hline
\end{tabular}

\section{Conditions in the differential diagnosis of COPD}

\section{Asthma}

Suggestive features-Family history, atopy, non-smoker, young age, nocturnal symptoms

Investigation-Peak flow monitoring, reversibility testing

\section{Congestive heart failure}

Suggestive features-Orthopnoea, history of ischaemic heart disease, fine lung crackles

Investigation-Chest radiograph, electrocardiogram, echocardiogram

Lung cancer

Suggestive features-Haemoptysis, weight loss, hoarseness

Investigation-Chest radiograph, bronchoscopy, computed tomography

\section{Bronchiectasis}

Suggestive features-Copious sputum production, frequent chest infections, childhood pneumonia, coarse lung crackles

Investigation-Sputum microscopy, culture, and sensitivity; high resolution computed tomography
Interstitial lung disease

Suggestive features-Dry cough; history of connective tissue disease; use of drugs such as methotrexate, amiodarone, etc; fine lung crackles

Investigation-Pulmonary function testing, chest radiograph, high resolution computed tomography, lung biopsy, test for autoantibodies

Opportunistic infection

Suggestive features-Dry cough, risk factors for immunosuppression, fever

Investigation-Chest radiograph; sputum microscopy, culture, and sensitivity; induced sputum; bronchoalveolar lavage

\section{Tuberculosi}

Suggestive features-Weight loss, haemoptysis, night sweats, risk factors for tuberculosis and immunosuppression

Investigation-Chest radiograph; sputum microscopy, culture, and sensitivity 


\section{Examination}

Because of the heterogeneity of COPD, patients may show a range of phenotypic clinical pictures. However, physical examination can be normal especially in patients with mild disease, although it may help suggest an alternative or coexistent diagnosis. Moreover, features of advanced airflow obstruction-peripheral and central cyanosis, hyperinflated chest, pursed lip breathing, accessory muscle use, wheeze, diminished breath sounds, and paradoxical movement of the lower ribs-are found in other chronic respiratory conditions and are therefore of low diagnostic sensitivity and specificity.

Cor pulmonale is defined as right ventricular hypertrophy due to any chronic lung disorder. Some patients with severe COPD may show signs consistent with cor pulmonale (raised jugular venous pressure, loud $\mathrm{P}_{2}$ heart sounds due to pulmonary hypertension, tricuspid regurgitation, pitting peripheral oedema, and hepatomegaly). Look for skeletal muscle wasting and cachexia, which may be present in those with advanced disease. Finger clubbing is not found in COPD, and its presence should prompt thorough evaluation to exclude a cause such as lung cancer, bronchiectasis, or idiopathic pulmonary fibrosis. At presentation, record the weight and height and calculate the body mass index (weight $(\mathrm{kg}) /($ height $\left.(\mathrm{m})^{2}\right)$ ). It is categorised for both men and women as $<18.5$ for underweight, 18.5-24.9 for normal, 25-29.9 for overweight, and $\geq 30$ for obese.

\section{Investigations}

\section{Peak expiratory flow}

Solitary peak expiratory flow readings can seriously underestimate the extent of airflow obstruction, while serial monitoring of peak expiratory flow is not generally useful in the diagnosis of COPD.

\section{Spirometry}

While history and examination are necessary in the diagnostic work up, showing airflow obstruction is vital in confirming the diagnosis. Spirometry should be arranged in all patients with suspected COPD. It is increasingly performed in primary care and can be carried out with minimal training for both clinician and patient. It measures

- Forced vital capacity (FVC)-the maximum volume of air forcibly exhaled after full inspiration (that is, from total lung capacity)

- Forced expiratory volume in one second $\left(\mathrm{FEV}_{1}\right)$-the volume of air exhaled during the first second of the FVC manoeuvre

- The $\mathrm{FEV}_{1} / \mathrm{FVC}$ ratio.

Compare the $\mathrm{FEV}_{1}$ and $\mathrm{FVC}$ with predicted normal values for age, height, and sex. These measures are often expressed as the percentage predicted as well as in absolute values in litres. Airflow obstruction is present if the $\mathrm{FEV}_{1} / \mathrm{FVC}$ ratio is $<0.7$ and the $\mathrm{FEV}_{1}$ is $<80 \%$ of the predicted value. A ratio of $\geq 0.7$ is either normal or suggestive of a restrictive ventilatory defect.

Spirometry also allows patients with COPD to be categorised according to severity, and enables monitoring of changes in lung function over time and the response to treatment. If spirometric values return to normal after treatment then clinically significant COPD is not present.

\section{Reversibility testing}

Patients with COPD used to be categorised by their response to a therapeutic trial of corticosteroids. However, it is now known that an improvement in lung function after a short course of oral corticosteroids usually fails to predict future benefit from

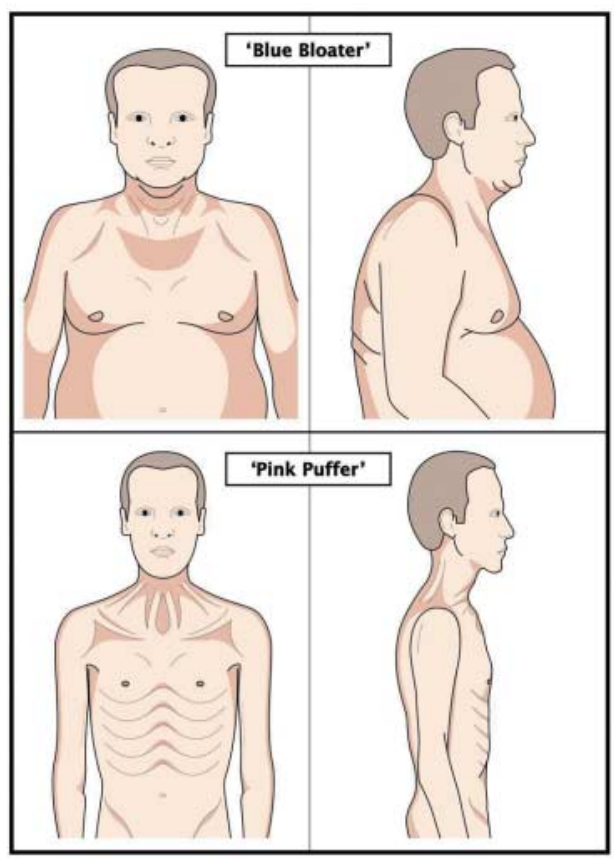

The clinical heterogeneity of COPD: a typical "blue bloater" (top) and "pink puffer" (bottom)

Common causes of obstructive and restrictive spirometry

Obstructive disorders

- COPD

- Neuromuscular disease $\bullet$ Interstitial lung disease $\bullet$ Kyphoscoliosis

- Previous - Pleural effusion $\quad$ Obesity

pneumonectomy

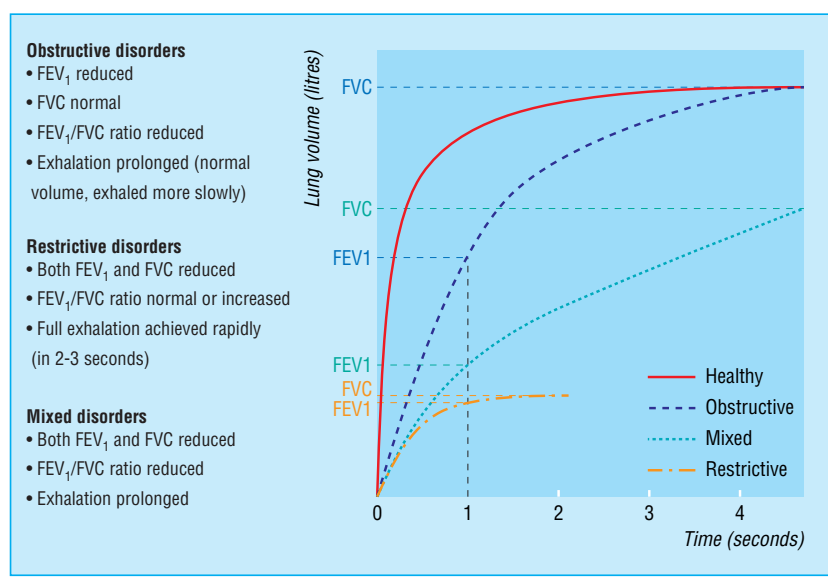

Volume time curves showing typical features of obstructive, restrictive, and mixed obstructive-restrictive ventilatory disorders

Categorisation of COPD severity by different guidelines

\begin{tabular}{lccc} 
& \multicolumn{3}{c}{$\boldsymbol{\%}$ of predicted FEV } \\
\cline { 2 - 4 } Airflow obstruction & BTS & ATS-ERS & GOLD \\
\hline Mild & $50-80 \%$ & $\geq 80 \%$ & $\geq 80 \%$ \\
\hline Moderate & $30-49 \%$ & $50-80 \%$ & $30-79 \%$ \\
\hline Severe & $<30 \%$ & $30-50 \%$ & $<30 \%$ \\
\hline Very severe & & $<30 \%$ & \\
\hline
\end{tabular}

$* \mathrm{FEV}_{1} / \mathrm{FVC}$ ratio should be $<0.7$ in all cases.

$\mathrm{BTS}=$ British Thoracic Society. ATS-ERS = American Thoracic Society and European Respiratory Society. GOLD = Global Initiative for Chronic Obstructive Lung Disease. The ATS/ERS guidelines also suggest an "at risk" group-smokers with typical symptoms of COPD with an $\mathrm{FEV}_{1} / \mathrm{FVC}$ ratio $>0.7$ and $\mathrm{FEV}_{1} \geq 80 \%$ predicted. The GOLD guidelines suggest an "at risk" category in smokers with typical symptoms of COPD but normal spirometry 
long term inhaled corticosteroids. Reversibility testing to oral corticosteroids or inhaled bronchodilator is not always necessary, but it should be performed if asthma is thought likely or if the response to treatment ( $\beta_{2}$ agonists or corticosteroids) is surprisingly good. However, asthma can often be distinguished from COPD by the history, examination, and baseline spirometry.

More detailed lung function measurements such as lung volumes (total lung capacity and residual volume), gas transfer coefficient, and walking distance in six minutes can be done if diagnostic doubt persists or more thorough evaluation is required.

\section{Imaging}

At the time of diagnosis, arrange a chest radiograph in all patients. This is useful in excluding other conditions as a cause of respiratory symptoms and may show typical features of COPD. When there is diagnostic doubt or a surgical procedure is contemplated (such as lung volume reduction surgery or bullectomy) computed tomography of the chest is required.

\section{Other tests}

A full blood count should be arranged. This may show secondary polycythaemia, which can result from chronic hypoxaemia, and it is important to exclude anaemia as a cause of breathlessness. A raised eosinophil count suggests the possibility of an alternative diagnosis such as asthma.

In patients with signs of cor pulmonale, electrocardiography may show typical changes of chronic right sided heart strain. However, echocardiography is more sensitive in detecting tricuspid valve incompetence, as well as right atrial and ventricular hypertrophy, and may indirectly assess pulmonary artery pressure. It is also useful in determining whether left ventricular dysfunction is present, especially when the spirometric impairment is disproportional to the extent of breathlessness. Ischaemic heart disease may be the sole or contributory cause of breathlessness even in the absence of chest pain, and investigations should be tailored accordingly. Indeed, dyspnoea due to causes other than COPD should be considered when the extent of physical limitation seems disproportionate to the degree of airflow obstruction, or in patients with normal oxygen saturation who have little or no fall in levels during a six minute walk.

Patients with a family history of COPD or in whom it presents at a young age (especially when smoking pack years are negligible) should have their $\alpha_{1}$ antitrypsin concentrations measured. If $\alpha_{1}$ antitrypsin deficiency is discovered, appropriate family screening and counselling is advised.

Pulse oximetry is useful in most patients, especially those with advanced disease (such as $\mathrm{FEV}_{1}<50 \%$ predicted) or polycythaemia, in order to check for significant hypoxaemia. Patients with a resting oxygen saturation of $<92 \%$ should have measurement of arterial blood gases and, where necessary and appropriate, be assessed for long term domiciliary or ambulatory oxygen therapy.

Graeme $\mathrm{P}$ Currie is specialist registrar and Joe $\mathrm{S}$ Legge is consultant in the Respiratory Unit, Aberdeen Royal Infirmary, Aberdeen.

The ABC of chronic obstructive pulmonary disease is edited by Graeme P Currie. The series will be published as a book by Blackwell Publishing in autumn 2006.

Competing interests: GPC has received funding for attending international conferences and honorariums for giving talks from pharmaceutical companies GlaxoSmithKline, Pfizer, and AstraZeneca.

BMJ 2006;332:1261-3

\section{Changes in lung function consistent with a diagnosis of asthma}

- Improvement in $\mathrm{FEV}_{1}$ by $\geq 15 \%$ and $\geq 200 \mathrm{ml}$ (or in peak expiratory flow of $\geq 20 \%$ and $\geq 60 \mathrm{l} / \mathrm{min}$ ) 20 minutes after taking $400 \mu \mathrm{g}$ (2 puffs) inhaled salbutamol or $2.5 \mathrm{mg}$ nebulised salbutamol

- Improvement in $\mathrm{FEV}_{1}$ by $\geq 15 \%$ and $\geq 200 \mathrm{ml}$ (or in peak expiratory flow of $\geq 20 \%$ and $\geq 60 \mathrm{l} / \mathrm{min}$ ) after taking $30 \mathrm{mg}$ oral prednisolone for two weeks

- Variability in peak expiratory flow (difference between highest and lowest scores) of $\geq 20 \%$ during three consecutive days over two weeks

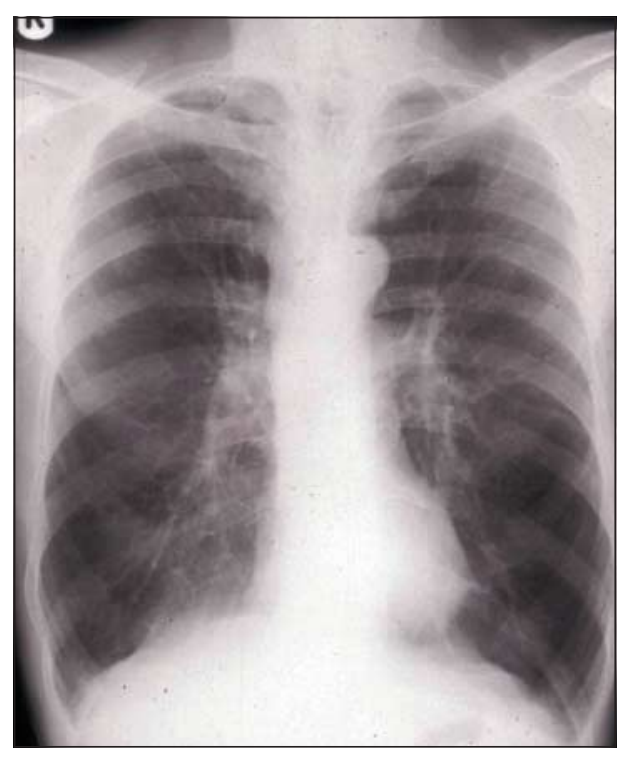

Chest radiograph showing typical changes of COPD (hyperinflated lung fields, flat diaphragms, prominent pulmonary arteries, increased translucency of lung fields and "squared off" lung apices)

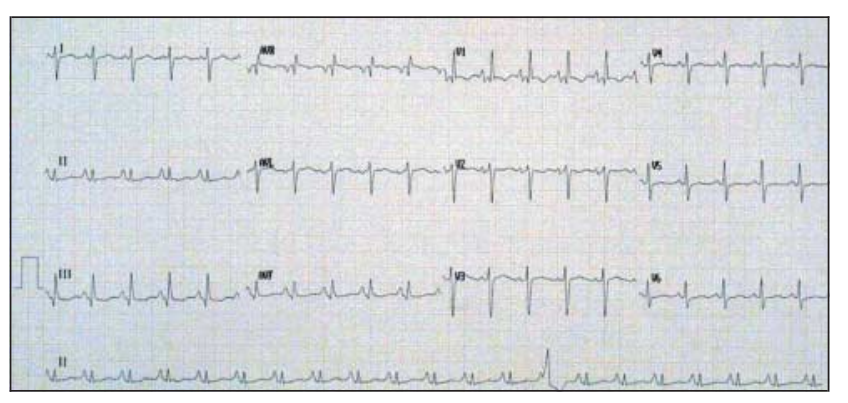

Electrocardiogram showing typical changes in a patient with cor pulmonale secondary to advanced COPD (p-pulmonale, right axis deviation, partial right bundle branch block, dominant $\mathrm{R}$ wave in lead $\mathrm{V} 1$ )

\section{Further reading}

- National clinical guideline on management of chronic obstructive pulmonary disease in adults in primary and secondary care. Thorax 2004;59(suppl 1):1-232.

- Celli BR, MacNee W. Standards for the diagnosis and treatment of patients with COPD: a summary of the ATS/ERS position paper. Eur Respir J 2004;23:932-46.

- Pauwels RA, Buist AS, Calverley PM, Jenkins CR, Hurd SS. Global strategy for the diagnosis, management, and prevention of chronic obstructive pulmonary disease. NHLBI/WHO Global Initiative for Chronic Obstructive Lung Disease (GOLD) Workshop summary. Am J Respir Crit Care Med 2001;163:1256-76.

- British guideline on the management of asthma. Thorax 2003;58 (suppl 1):i1-94.

- Pauwels RA, Rabe KF. Burden and clinical features of chronic obstructive pulmonary disease (COPD). Lancet 2004;364:613-20. 